\title{
Hydrogel Microneedle Arrays for Transdermal Drug Delivery
}

\author{
Xiaoyun Hong ${ }^{1}$, Zaozhan $\mathrm{Wu}^{1}$, Lizhu Chen ${ }^{1}$, Fei $\mathrm{Wu}^{1, *}$, Liangming $\mathrm{Wei}^{2, *}$, Weien Yuan ${ }^{1, *}$
}

(Received 21 November 2013; accepted 10 March 2014; published online July 1, 2014)

\begin{abstract}
Stratum corneum is the main obstacle for drugs to pass through the skin. Microneedles are composed of arrays of micro-projections formed with different materials, generally ranging from 25-2000 $\mu \mathrm{m}$ in height. Microneedles straightly pierce the skin with its short needle arrays to overcome this barrier. Microneedles can be divided into several categories, for instance, solid microneedles, coated microneedles, and hollow microneedles and so on. However, all these types have their weak points related to corresponding mechanisms. In recent years, pioneering scientists have been working on these issues and some possible solutions have been investigated. This article will focus on the microneedle arrays consisting of hydrogels. Hydrogels are commonly used in drug delivery field. Hydrogel microneedles can be further divided into dissolving and degradable microneedles and phase transition microneedles. The former leaves drug with matrix in the skin. The latter has the feature that drugs in the matrix are delivered while the remaining ingredients can be easily removed from the skin after usage. For drugs which are required to be used every day, the phase transition microneedles are more acceptable. This article is written in order to summarize the advantages of these designs and summarize issues to be solved which may hinder the development of this technology.
\end{abstract}

Keywords: Hydrogel; Microneedle; Phase transition; Dissolving and degradable

Citation: Xiaoyun Hong, Zaozhan Wu, Lizhu Chen, Fei Wu, Liangming Wei and Weien Yuan, "Hydrogel Microneedle Arrays for Transdermal Drug Delivery", Nano-Micro Lett. 6(3), 191-199 (2014). http://dx.doi. org/10.5101/nml140021r

\section{Introduction}

Microneedle (MN) technology, a minimally invasive drug delivery system, has the potential to be an alternative to hypodermic needle tech [1]. Generally, MNs are composed of arrays of micro-projections formed with different materials, generally ranging from 25-2000 $\mu \mathrm{m}$ in height [2], with different tip shapes and tip intervals, being attached to a base support [3]. When applied to skins, they puncture the epidermis and reach the dermis. Since the depths of needle insertion is within the non-innervated layer of the skin, this technique would allow painless delivery and improve patient compliance [4]. This kind of structures help to overcome the weaknesses of traditional transdermal delivery sys- tems, which only allow small and lipophilic molecules to be delivered by passive diffusion, while keeping the strengths of accessibility, safety, painless drug administration, potential for self-administration, and avoidance of enzymatic degradation in the gastrointestinal tract or liver as well as needle phobia [5-7].

Hydrogels are polymers with a three-dimensional structure that exhibit the ability to swell in water and keep significant amount of water within the structures [8]. They can be divided into natural hydrogels and synthetic ones. Natural hydrogels include agarose, methylcellulose, hyaluronic acid, chitosan, starch, collagen and other naturally derived polymers while synthetic ingredients include polyvinyl alcohol, sodium polyacrylate, acrylate polymers and copolymers with an abun-

\footnotetext{
${ }^{1}$ School of Pharmacy, Shanghai Jiao Tong University, Shanghai 200240, China

${ }^{2}$ Research Institute of Micro/Nano Science and Technology, Shanghai Jiao Tong University, Shanghai 200240, China

*Corresponding authors. E-mail: yuanweien@126.com; feiwu@sjtu.edu.cn; lmwei@sjtu.edu.cn
} 
dance of hydrophilic groups [9]. Additionally, hydrogels that do not exhibit any significant volume transition to environmental changes $(\mathrm{pH}$, temperature, photo field, ion concentration, composition of the solvent, etc.), which are generally obtained from hydrophilic monomers, are defined as "conventional" hydrogels, while others, which can change their volumes abruptly in response to the changes of the external environmental factors are classified as "intelligent" hydrogels [10-13].

\section{Problems of non-hydrogel MNs}

There are several kinds of MNs, namely, solid MNs for skin pretreatment to increase skin permeability, MNs coated with drugs, hollow MNs for drug infusion into the skin, polymeric or polysaccharide MNs that encapsulate drugs and partially or fully dissolve in the skin [14-17].

Solid MNs, which do not contain drugs themselves, enhance the permeability of drugs, for instance, small molecules, proteins and nanoparticles, by creating pores into the skins [18]. They are the first generation of MNs and generally fabricated with silicon or metals [19]. However, this requires a two-step application, which is not convenient for patients. Skin is a self-regenerating organ which heals itself [5] and the different recovery rates caused by individual differences significantly affect the bioactivity administration of drugs. In order to retard the recovery of skin, some additional drugs are applied to the skin including diclofenac sodium. Brogden et al. [20] suggested that the micro-pore lifetime could be prolonged with simple topical administration of a non-specific cyclooxygenase inhibitor. Ghosh et al. [5] utilized naltrexone (NTX) with diclofenac co-drugs for systemic delivery of NTX into the treated skin and the results indicated that the co-drug approach could be used for the development of a 7-day transdermal system. These results showed the possibility for better delivery of drugs after the skins were treated with solid MN-enhanced delivery, thus expanding the transdermal field to a wider variety of clinical conditions. On the other hand, if some of the needles happen to break and are left in the skin, irritation is inevitable. The fabrication cost is high and the disposition of wastes is also a question. Some materials, for example, silicon, require clean room processing and are not FDA-approved biomaterials.

Drug coated solid MNs was a simple, versatile, and controllable method to coat microneedles with DNA, proteins, microparticles and viruses for rapid delivery into the skin [21], which solve the problem of two-step application. Moreover, the drug coated MNs may also combined with hydrogel. M Pearton et al. [22] showed the ability of micro-fabricated needles to create some channels and a mediate reporter gene expression in vi- able human skin by using pDNA loaded the hydrogels. This is another kind of drug coating. The skin was first treated with pDNA loaded hydrogels and then punctured with MNs. Further in vivo studies with these formulations will research the ability of these systems controlled-release pDNA expression. But the results showed that the total number of positive expression was less than solution prescription in $24 \mathrm{~h}$. However, due to the design, the drug can only be loaded onto the surface of MNs, so the drug loading amount is relatively low [23-25]. The cost of fabrication is also another concern.

Hollow MNs usually require very precise and high cost manufacturing technology, which prevent them from large scale production [26]. In this approach, large scale reproduction with low costs is pursued sometimes based on self-assembly and molding of soft materials. Polydimethylsiloxane (PDMS) is an elastomer used in micro fabrication, but it is to some extent with low stiffness. However, Matteucci et al. [27] investigated re-usable poly vinyl alcohol (PVA) masters, which were found to be easily obtainable and perform better in fabrication of needle pillars where aspect ratios higher than five are needed. PVA masters also show advantages of higher stiffness, hydrophilic behavior and resistance to poly methyl methacrylate (PMMA) developers for the fabrication of PMMA structures. However, if hollow MNs are occasionally broken in the skin, significant leakage or uncontrolled drug release may occur [2]. There are also risks that the body tissue blocks the narrow channels which interfering the drug dosage.

\section{The advantages of hydrogel MNs}

Hydrogel MNs are one kind of polymeric or polysaccharide MNs according to the definition of hydrogel. Besides the hollow MNs which are fabricated with polymers or the hydrogel coated the surfaces of solid MNs, there are three kinds of drug-loading methods. Some MNs only have drugs in the tips; some in the patches; others have drugs in both of them. All of these methods provide large spaces for drugs to be loaded. The drug loading amount is better than the solid MNs and drug coated MNs as well as the hollow MNs. Polymeric and polysaccharide MNs include a variety of MNs with different characters. Some polymers and polysaccharides are drawing increasing attentions because of their excellent biocompatibility, degradability, and nontoxicity $[28,29]$. Polymer microneedles also offer the benefits of ease of fabrication, cost-effectiveness, and the capacity for mass production, as well as controlled drug release with the help of water solubility and degradation properties of polymers [30]. Hydrogel MNs are overall easier and more economical to be fabricated from materials which are more likely to be biocompatible and with 
FDA approvals. The fabrication methods often include the photolithographic process and micro-molding process [30]. For micro-molding processes which are lower cost, centrifugation and vacuum method have been developed to obtain polymer microneedles with the proper shapes; it is obvious that the latter is more suitable for mass production of MNs. Thus there is a new research field to find an inexpensive, easy to be manufactured, waterproof while air permeable mold to achieve mass production of MNs. According to the function mechanism of hydrogel MNs, they are divided into two categories.

The first is dissolving or degradable MNs. They bear common features in the mechanism that the drug release pattern is related to the dissolution or degradation of the MN matrix, i.e. the polymer or polysaccharide themselves [30-32]. These MNs dissolve or degrade in the skin and thereby leave no sharp medical waste after use [33-35]. The common process is shown in Fig. 1. Jeong W. Lee et al. [36] applied FDA-approved ultralow viscosity carboxymethylcellulose and amylopectin to present a dissolving $\mathrm{MN}$ design involving preparation under mild conditions that may be suitable for protein delivery.

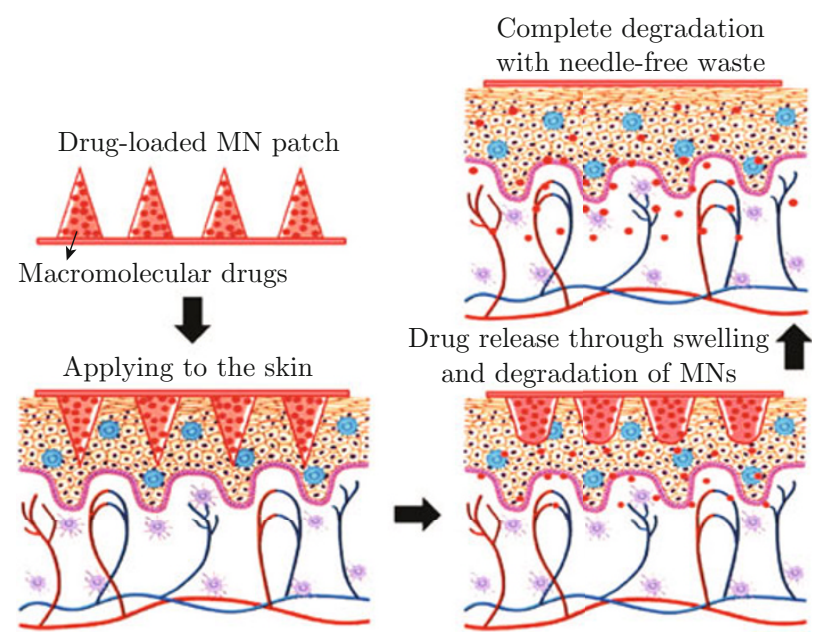

Fig. 1 The common action mechanism of degradable microneedles [39].

Dissolving microneedles can dissolve within the skin safely and need not be removed from the patient's skin when applied. After the insertion to the skin, dissolving microneedles dissolve quickly and release the loaded drugs in a short time. Ming-Hung Ling et al. [37] presented a dissolving microneedle patch, which is composed of starch and gelatin and carried insulin as the model drug. Regarding the in vitro drug release test, the dissolving microneedles released almost all the insulin carried into the skin within 5 minutes. Consistent with the in vitro skin insertion test, the starch/gelatin microneedles dissolved completely within 5 minutes after insertion into the porcine skin. The dissolving mi- croneedles have enough mechanical strength and stable encapsulation of bioactive molecules, which is promising for the transdermal delivery.

Biodegradable MNs degrade in the skin after application, whereby the release of incorporated drugs can be sustained up to months by choosing the proper polymers. Mei-Chin Chen et al. [38-39] investigated that drugs loaded in chitosan carriers can be released through swelling and degradation of the chitosan matrix, leading to a clear sustained-release effect. Chitosan with suitable molecular weight can be cleared by the kidney in vivo, whereas excessive molecules can be cleaved by proteases into fragments to be suitable for renal clearance. They used OVA as a model antigen to be loaded in the microneedles. The release profile result showed an initial burst within one day and a slow release followed for at least 7 days, with almost $90 \%$ of the OVA released. The in vivo degradation of VOA-loaded chitosan microneedles also showed that even 14 days after application, small residual fragments of the microneedle still can be found. The slow degradation rate demonstrated that the embedded chitosan microneedles degrade gradually and may lead to a sustained antigen release.

Some biodegradable MNs leave the needles in skin to deliver bolus drugs or sustained release of drugs by separating the needles from the patches in the skin. Min Kim et al. [40] demonstrated MN separation mediated by hydrogel swelling in response to contact with body fluid. In such cases, the tips of biodegradable polymeric MNs were separated because of hydrogel microparticles, which were fabricated between the needle tips and the patches, expand quickly and lose mechanical strength rapidly by swelling and absorbing body fluid. Besides, there were also hydrogel MNs combined with metal shafts to form biodegradable ones. Leonard Y. Chu et al. [41] investigated separable arrowhead MNs which upon insertion in the skin, the sharp-tipped polymer arrowheads induced hydrogel part which contained drugs to separate from their metal shafts. Due to rapid separation of the arrowhead tips from the shafts within seconds, administration using arrowhead MNs can be performed rapidly, while the release kinetics profiles of drug can be independently controlled based on separable arrowhead formulation.

The second is phase transition MNs in which drug release as a result of the polymer swelling when absorbing body fluid. This kind of MNs leaves few or no residuals after application. These MNs preserve the advantages of other MNs, such as the drug permeating amount and rate improvement, large drug loading amount and relatively easy to be fabricated. They also have the potential to be daily used since few non-drug residuals will be left in the skin, which may increase the patients' compliance. They are so far a very promising MN technologies. Ryan F. Donnelly et al. [42,43] 
developed MNs made of Gantrez ${ }^{\circledR}$ AN-139, a copolymer of methyl vinyl ether and maleic anhydride, which could be removed completely and intact from the skin. The needle tips swell in skin to produce continuous, unblockable conduits from patch-type drug reservoirs to the dermal microcirculation, thus allowing prolonged transdermal drug administration. According to their findings, delivery of macromolecules was no longer limited to what can be loaded into the MNs themselves and transdermal delivery drug was controlled by using the crosslink density of the hydrogel system rather than the stratum corneum. The materials they chose were biocompatible, non-irritant and raised no safety concerns in human volunteers. The MNs can be fabricated in a wide range of patch sizes and MN geometries by adjusting the molds used. Furthermore, they can also be easily sterilized. It is reported that Young's modulus and hardness of non-toxic Gantrez ${ }^{\circledR}$ AN-139 MN were noted as $6.56 \pm 0.56 \mathrm{GPa}, 385.6 \pm 12.00 \mathrm{MPa}$, respectively, which indicates it as a stiffness microneedle base material [44]. PVA is a well-known polymer, which can generate hydrogels by physical or chemical crosslinking and is applied in the field of controlled drug release $[45,46]$. Sixing Yang et al. $[47,48]$ developed PVA MNs, which compared to other hydrophilic MNs, would not dissolve in the skin, but swell and therefore diffusion channels were opened for drug release. The microneedles were adequately swollen after embedding into human skin and were strong enough to penetrate skin. The results showed that the MNs were embedded reliably into the skin using a force of about $5 \mathrm{~N}$ with one thumb. The easy application method is also helpful for the self-administration of patients.

Biodegradable polymeric polylactic-co-glycolic acid (PLGA) MNs and dissolvable MNs, for instance, sodium alginate MNs are proved to create micro-holes in the skin layers without breaking, although the former one have more resistance and mechanical stability $[49,50]$.

MNs could be combined with other methods such as electroporation and iontophoresis (ITP) to enhance the delivery of drugs [51-53]. Electroporation is the transitory structural perturbation of lipid bilayer membranes due to the application of high voltage pulses. Its application to the skin has been shown to increase transdermal drug delivery by several orders of magnitude [54]. Iontophoresis was often used combined with hydrogel MNs, which was reported to broaden the range of drugs suitable for transdermal delivery, as well as enabling the rate of delivery to be achieved with precise electronic control [55]. Martin J. Garland et al. [51] found that whilst the combination of MN and ITP did not further enhance the extent of small molecular weight solute delivery, the extent of peptide/protein release was significantly enhanced when ITP was used in combination of the soluble PMVE/MA MN arrays.
Micro- and nanoparticles were also applied. CherngJyh Ke et al. [56] managed co-delivering of two model drugs trans-dermally, Alexa 488 and Cy5, in sequence, based on a system of polyvinylpyrrolidone (PVP) MNs that contain $\mathrm{pH}$ responsive poly(D,L-lactic-co-glycolic acid) hollow microspheres (PLGA HMs). The key component of this system was $\mathrm{NaHCO}_{3}$, which could be easily incorporated into HMs. After HMs are treated with an acidic solution (simulating the skin $\mathrm{pH}$ environment), a large number of $\mathrm{CO}_{2}$ bubbles were formed to generate pressure inside the HMs and creates pores inside their PLGA shells, releasing the encapsulated Cy5 (as shown in Fig. 2).

\section{Existing problems of hydrogel MNs}

Whilst hydrogel MNs are more promising technologies over non-hydrogel ones, they also have problems to be solved. Firstly, although the dissolving or degradable MNs comparatively carry more drug amounts, the matrix, which accounted for a large portion of the needles, dissolve or degrade in the skin. The process is not the best choice since the MNs are transdermal administration methods. However, since the matrixes dissolve or degrade in skins, this kind of MNs is not suitable for drugs which require daily administration. The retention of matrix in the skin will cause low patients compliance and other potential side effects if the drugs are applied every day or within short intervals through MNs. The most suitable active ingredients to be loaded are vaccines, for they are used only once or just several times and thus the amount of matrixes deposit in skin will be much more acceptable.

As mentioned above, scientists developed phase transition MNs in order to solve this problem. Poly methyl vinyl ether/ maleic acid (PMVE/MA) and poly (ethylene glycol) 10,000 (PEG) were utilized by Donnelly et al. [42] to fabricate MN. The diffusion of body fluid caused controlled swelling of the MN arrays to form an in situ hydrogel conduit (as shown in Fig. 3). This further resulted in liberation and diffusion of drug molecules from the adhesive patch through the hydrogel MN into the skin. The striking feature was that the hydrogel $\mathrm{MN}$ arrays remained intact, even after removal from the skin, thereby leaving no polymeric material in the skin following drug delivery. They developed adhesive patches, containing the molecules at defined loadings, were then attached to the upper base plates of hydrogel-forming MN, with the novel composite system termed as "integrated MN". However, according to the experimental details, for example, the delivery of insulin from the MN (with $600 \mu \mathrm{m}$ height, $300 \mu \mathrm{m}$ width at base, $50 \mu \mathrm{m}$ interspacing, \& $19 \times 19$ arrays, i.e., about $0.45 \mathrm{~cm}^{2}$ ) was investigated in a diabetic Sprague-Dawley rat model. Positive controls were 


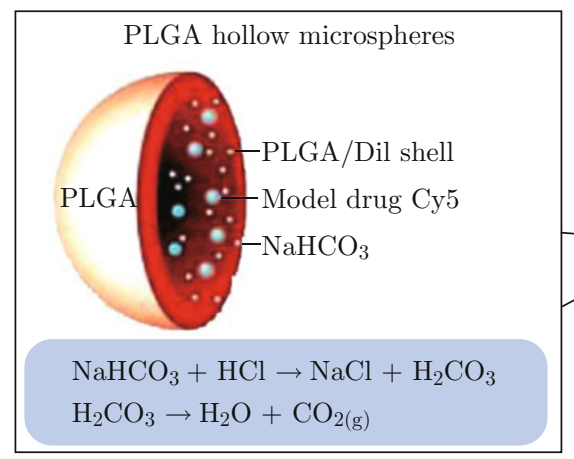<smiles>C=C(CO[C+](C)(C)C)C(=O)OCC(C)=O</smiles>

PLGA Insertion of microneedles

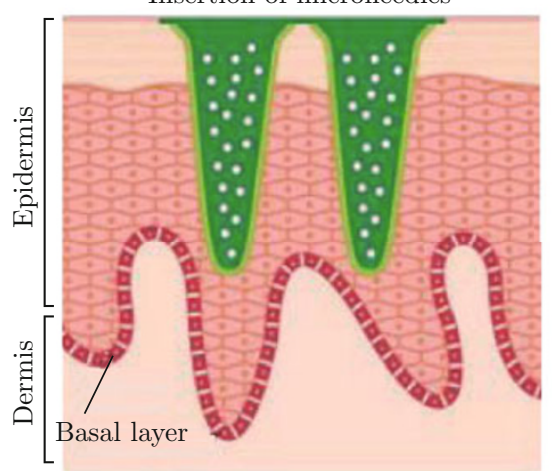

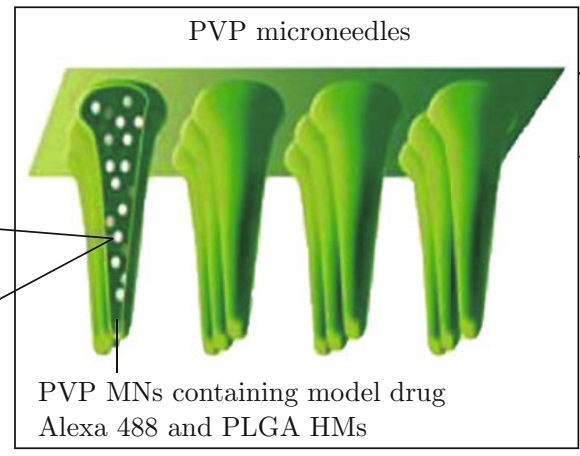

Alexa 488 and PLGA HMs
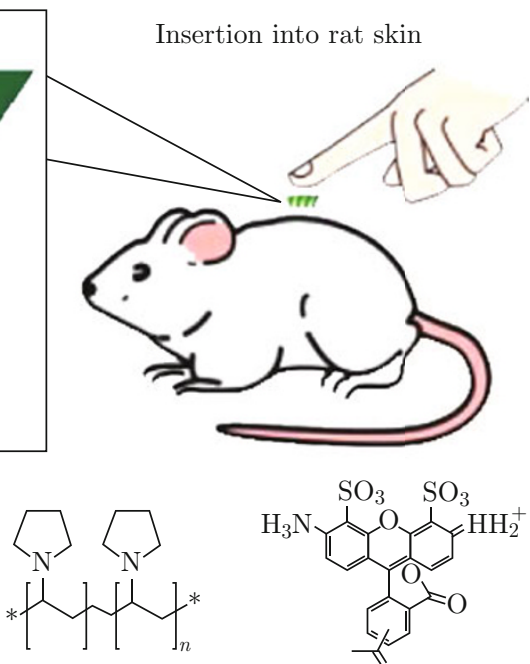

PVP

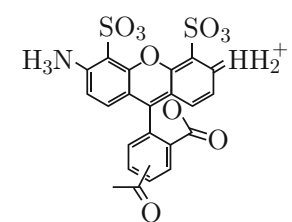

Alexa 488
$\mathrm{Cy} 5$

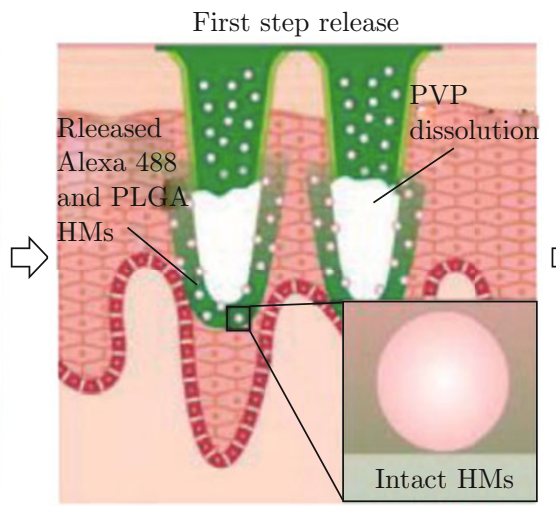

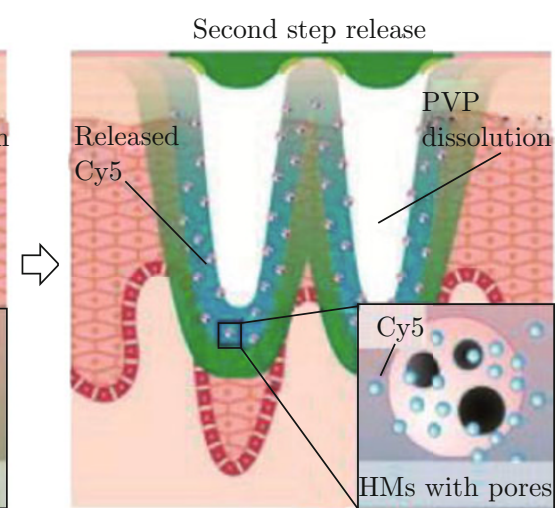

Fig. 2 The design of PVP MN microneedles. pH-responsive PLGA HMs and their mechanism for two model drugs Cy5 and Alexa 488 in sequence transdermal. After the HMs were inserted into skin, the first Alexa 488 rapid release and HMs using Dil-labeled were accomplished because the PVP quickly dissolve. And then the second Cy5 release was stimulated by using the acidic environment of skin. PLGA HMs:poly(DL-lactic-coglycolic acid) hollow microspheres;PVP MNs:polyvinylpyrrolidone microneedles [56].

performed by subcutaneously injecting bovine insulin solution in PBS pH 7.4 at a dose of 0.2 IU per animal. The integrated MN was at a loading of $5 \mathrm{mg} / \mathrm{cm}^{2}$ insulin, which equaled to about $2.2 \mathrm{mg}$ or more than 60 IU insulin. The dose in the MNs was over 300 times comparable to that of the positive control. The results showed that the blood glucose level (BGL) dropped to about $90 \%$ of its original level within $2 \mathrm{~h}$ and fell further to about $37 \%$ by the end of the $12 \mathrm{~h}$ experimental period if the MNs were applied alone; Even the combination of integrated hydrogel MN and anodal ITP (applied for a $2 \mathrm{~h}$ period, after which the electrodes and $\mathrm{MN} /$ insulin patch were removed) led to a rapid reduction in BGL, dropping to approximately $47 \%$ within $2 \mathrm{~h}$ and $32 \%$ within $6 \mathrm{~h}$ compared with the positive control. According to the doses in the MNs, the bioactivity of this method was quite unsatisfactory. The combination of MN and anodal ITP led to the increase of MN cost and inconvenience of drug administration by patients themselves. The decrease of BGL level in the first 2 $\mathrm{h}$ period by $\mathrm{MN}$ alone was insignificant, which meant that it could not release sufficient drugs in a short period of time.

The phase transition PVA MNs developed by Sixing Yang et al. [46] was made with the application of the purple-sand female mold. Purple sand was traditionally used by Chinese to make teapots [57,58]. Although this mold had the advantage of being air permeable but water resistant [59], and in coordination with a vacuum on the opposite side, it perfectly met the demand of hydrophilic microneedle patch preparation. However, since purple sand was hydrophilic, the concentration of the polymeric solution might change during the fabrication process when a vacuum was applied. The purple sand absorbed the water in the polymeric solution and it was hard to control the concentration, as well as the drug loading amount. The MN was $0.8 \mathrm{~mm}$ in length and $0.3 \mathrm{~mm}$ in base, while the length inserted into skin was $0.4 \mathrm{~mm}$. It can be calculated that the volume inserted into skin was only $12.5 \%$ of the total 


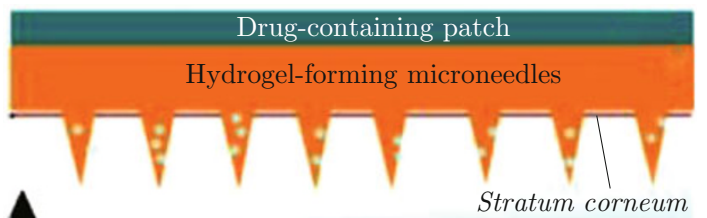

Viable epidermis
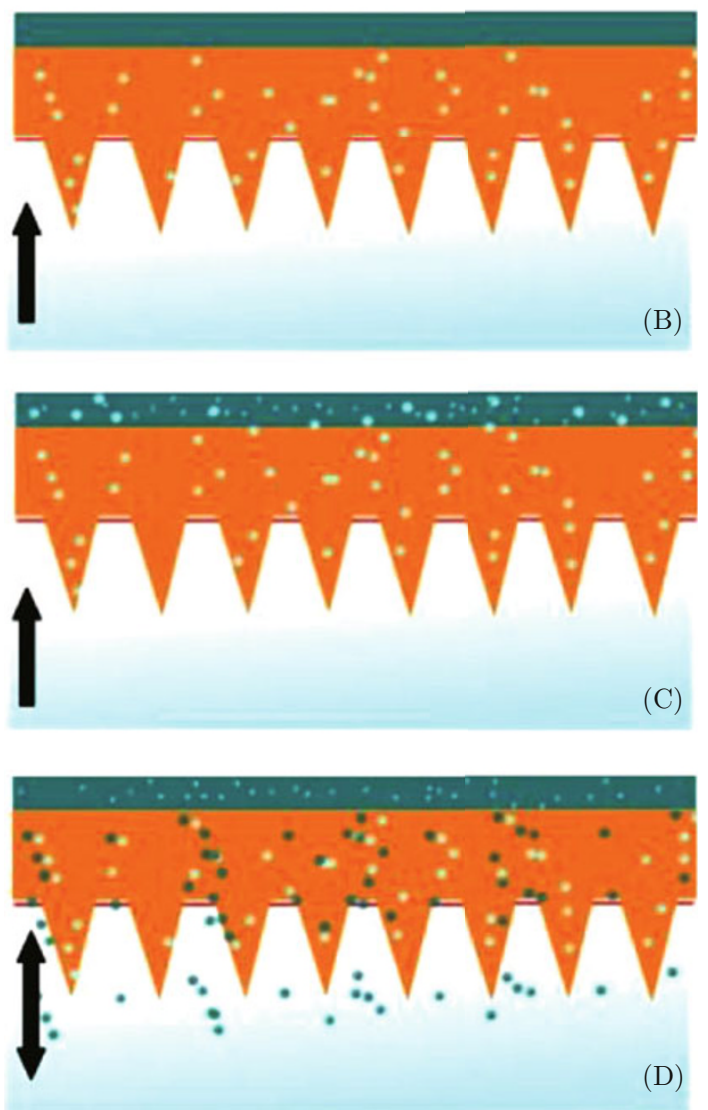

Fig. 3 The design of hydrogel-forming MNs [60].

needle tip. This should be improved in order to achieve better release rate and amount of drugs.

Ryan F. Donnelly and Juntanon et al. [60-63] realized that MNs had the possibility to carry microorganisms into the skin and did related tests which indicated that microorganisms could penetrate skin beyond the stratum corneum following microneedle puncture. The numbers of microorganisms crossing the stratum corneum following microneedle puncture were greater than $10^{5} \mathrm{cfu}$ in each case while no microorganisms crossed the epidermal skin. When using a $21 \mathrm{G}$ hypodermic needle, more than $10^{4}$ microorganisms penetrated into the viable tissue and $10^{6} \mathrm{cfu}$ of Candida albicans and Staphylococcus epidermis completely crossed the epidermal skin in $24 \mathrm{~h}$. However, the MN applied in the test was shorter than $280 \mu \mathrm{m}$. In a variety of cases, the needle tips were longer than $500 \mu \mathrm{m}$, so the experiment did not cover this part. The anti-microorganism effect should be investigated further.

Overall, the hydrogel microneedles are more promising compared with their solid or hollow counterparts. There are also some weaknesses related to the dissolving or biodegradable mechanisms. The most prospective MN type is the hydrogel MN which does not dissolve or degrade in skin but with a controlled or sustained release of drugs. The application of other methods, including ITP or electroporation, may enhance the drug release rate, but they also increase the costs of MNs while lower the patients' compliance.

\section{Acknowledgments}

The study was supported by the Projects of National Science Foundation of China (No. 81373366 and 81173001), and Funds for Interdisciplinary Projects of Medicine and Engineering by Shanghai Jiao Tong University (No. YG2013MS52 and YG2013MS62).

\section{References}

[1] M. R. Prausnitz and R. Langer, "Transdermal drug delivery", Nat. Biotechnol. 26(11), 1261-1268 (2008). http://dx.doi.org/10.1038/nbt. 1504

[2] R. F. Donnelly, T. R. R. Singh and A. D. Woolfson, "Microneedle-based drug delivery systems: microfabrication, drug delivery, and safety", Drug Deliv. 17(4), 187-207 (2010). http://dx.doi.org/10.3109/ 10717541003667798

[3] Y. A. Gomaa, "Effects of microneedle length, density, insertion time and multiple applications on human skin barrier function: assessments by transepidermal water loss", Toxicol. In Vitro 24(7), 1971-1978 (2010). http://dx.doi.org/10.1016/j.tiv.2010.08.012

[4] S. P. Davis, B. J. Landis, Z. H. Adams, M. G. Allen and M. R. Prausnitz, "Insertion of microneedles into skin: measurement and prediction of insertion force and needle fracture force", J. Biomech. 37(8), 1155-1163 (2004). http://dx.doi. org/10.1016/j.jbiomech. 2003.12.010

[5] P. Ghosh, R. Pinninti, D. Hammell, K. Paudel and A. Stinchcomb, "Development of a codrug approach for sustained drug delivery across microneedle a treated skin", J. Pharm. Sci. 102(5), 1458-1467 (2013). http://dx.doi.org/10.1002/jps. 23469

[6] K. van der Maaden, W. Jiskoot and J. Bouwstra, "Microneedle technologies for (trans) dermal drug and vaccine delivery", J. Controlled Release 161(2), 645655 (2012). http://dx.doi.org/10.1016/j.jconrel. 2012.01 .042

[7] Y.-C. Kim, J.-H. Park and M. R. Prausnitz, "Microneedles for drug and vaccine delivery", Adv. Drug Deliver. Rev. 64(14), 1547-1568 (2012). http://dx. doi.org/10.1016/j.addr.2012.04.005

[8] T. Fan, M. Li, X. Wu, M. Li and Y. Wu, "Preparation of thermoresponsive and $\mathrm{pH}$-sensitivity 
polymer magnetic hydrogel nanospheres as anticancer drug carriers", Colloids Surface B: Biointerface 88(2), 593-600 (2011). http://dx.doi.org/10.1016/ j.colsurfb. 2011.07 .048

[9] C. J. Martin, C. J. Allendera, K. R. Braina, A. Morrisseyb and J. C. Birchall, "Low temperature fabrication of biodegradable sugar glass microneedles for transdermal drug delivery applications", J. Controlled Release 158(1), 93-101 (2012). http://dx.doi.org/10.1016/ j.jconrel.2011.10.024

[10] L. Liu, X. Tang, Y. Wang and S. Guo, "Smart gelation of chitosan solution in the presence of $\mathrm{NaHCO}_{3}$ for injectable drug delivery system", Int. J. Pharm. 414(1-2), 6-15 (2011). http://dx.doi.org/10.1016/ j.ijpharm. 2011.04.052

[11] K. M. Gattás-Asfura, E. Weisman, F. M. Andreopoulos, M. Micic, B. Muller, S. Sirpal, S. M. Pham and R. M. Leblanc, "Nitrocinnamate-functionalized gelatin: synthesis and "smart" hydrogel formation via photo-cross-linking", Biomacromolecules 6(3), 15031509 (2005). http://dx.doi.org/10.1021/bm049238w

[12] J. Kopeček, "Hydrogel biomaterials: a smart future?", Biomaterials 28(34), 5185-5192 (2007). http://dx. doi.org/10.1016/j.biomaterials . 2007.07.044

[13] I. Y. Galaev and B. Mattiasson, "Smart'polymers and what they could do in biotechnology and medicine", Trends Biotechnol. 17(8), 335-340 (1999). http://dx. doi .org/10.1016/S0167-7799(99)01345-1

[14] F. Wu, S. Yang, W. Yuan and T. Jin, "Challenges and strategies in developing microneedle patches for transdermal delivery of protein and peptide therapeutics", Curr. Pharm. Biotechno. 13(7), 1292-1298 (2012). http://dx.doi.org/10.2174/138920112800624319

[15] J.-H. Park, M. G. Allen and M. R. Prausnitz, "Polymer microneedles for controlled-release drug delivery", Pharm. Res. 23(5), 1008-1019 (2006). http://dx.doi. org/10.1007/s11095-006-0028-9

[16] L. Wei-Ze, H. Mei-Rong, Z. Jian-Ping, Z. YongQiang, H. Bao-Hua, L. Ting and Z. Yong, "Supershort solid silicon microneedles for transdermal drug delivery applications", Int. J. Pharm. 389(1), 122129 (2010). http://dx.doi.org/10.1016/j.ijpharm. 2010.01 .024

[17] L. Daugimont, N. Baron, G. Vandermeulen, N. Pavselj, D. Miklavcic, M.-C. Jullien, G. Cabodevila, L. M. Mir and V. Préat, "Hollow microneedle arrays for intradermal drug delivery and DNA electroporation", J. Membrane Biol. 236(1), 117-125 (2010). http://dx.doi. org/10.1007/s00232-010-9283-0

[18] Y. Qiu, G. Qin, S. Zhang, Y. Wu, B. Xu and Y. Gao, "Novel lyophilized hydrogel patches for convenient and effective administration of microneedlemediated insulin delivery", Int. J. Pharm. 437(1-2), 5156 (2012). http://dx.doi.org/10.1016/j.ijpharm. 2012.07.035

[19] L. Lin and A. P. Pisano, "Silicon-processed microneedles", J. Microelectromech. S. 8(1), 78-84 (1999). http://dx.doi.org/10.1109/84.749406
[20] N. K. Brogden, M. Milewski, P. Ghosh, L. Hardi, L. J. Crofford and A. L. Stinchcomb, "Diclofenac delays micropore closure following microneedle treatment in human subjects", J. Controlled Release 163(2), 220229 (2012). http://dx.doi.org/10.1016/j.jconrel. 2012.08 .015

[21] H. S. Gill and M. R. Prausnitz, "Coated microneedles for transdermal delivery", J. Controlled Release 117(2), 227-237 (2007). http://dx.doi.org/10.1016/ j.jconrel.2006.10.017

[22] M. Pearton, C. Allender, K. Brain, A. Anstey, C. Gateley, N. Wilke, A. Morrissey and J. Birchall, "Gene delivery to the epidermal cells of human skin explants using microfabricated microneedles and hydrogel formulations", Pharmaceut. Res. 25(2), 407-416 (2008). http://dx.doi.org/10.1007/s11095-007-9360-y

[23] A. K. Andrianov, A. Marin and D. P. DeCollibus, "Microneedles with intrinsic immunoadjuvant properties: microfabrication, protein stability, and modulated release", Pharmaceut. Res. 28(1), 58-65 (2010). http: // dx. doi.org/10.1007/s11095-010-0133-7

[24] A. Vrdoljak, M. G. McGrath, J. B. Carey, S. J. Draper, A. V. S. Hill, C. O'Mahony, A. M. Crean and A. C. Moore, "Coated microneedle arrays for transcutaneous delivery of live virus vaccines", J. Controlled Release 159(1), 34-42 (2012). http://dx.doi.org/10.1016/j . jconrel.2011.12.026

[25] M. Pearton, V. Saller, S. A. Coulman, C. Gateley, A. V. Anstey, V. Zarnitsyn and J. C. Birchall, "Microneedle delivery of plasmid DNA to living human skin: formulation coating, skin insertion and gene expression", J. Controlled Release 160(3), 561-569 (2012). http:// dx.doi.org/10.1016/j.jconrel.2012.04.005

[26] M. Ashraf, S. Tayyaba, A. Nisar, N. Afzulpurkar, D. Bodhale, T. Lomas, A. Poyai and A. Tuantranont, "Design, fabrication and analysis of silicon hollow microneedles for transdermal drug delivery system for treatment of hemodynamic dysfunctions", Cardiovasc. Eng. 10(3), 91-108 (2010). http://dx.doi.org/10. 1007/s10558-010-9100-5

[27] M. Matteucci, M. Fanetti, M. Casella and F. Gramatica, "Poly vinyl alcohol re-usable masters for microneedle replication", Microelectron. Eng. 86(46), 752 (2009). http://dx.doi.org/10.1016/j.mee. 2009.01 .068

[28] L. Y. Chu, S. O. Choi and M. R. Prausnitz, "Fabrication of dissolving polymer microneedles for controlled drug encapsulation and delivery: bubble and pedestal microneedle designs", J. Pharm. Sci. 99(10), 4228-4238 (2010). http://dx.doi.org/10.1002/jps. 22140

[29] X. Hong, L. Wei, F. Wu, Z. Wu, L. Chen, Z. Liu and W. Yuan, "Dissolving and biodegradable microneedle technologies for transdermal sustained delivery of drug and vaccine", Drug Des. Dev. Ther. 7, 945-952 (2013). http://dx.doi.org/10.2147/DDDT . S44401

[30] J. W. Lee, M.-R. Han and J.-H. Park, "Polymer microneedles for transdermal drug delivery", J. Drug Target 21(3), 211-223 (2013). http://dx.doi.org/10. 3109/1061186X . 2012.741136 
[31] K. Migalska, D. I. J. Morrow, M. J. Garland, R. Thakur, A. D. Woolfson and R. F. Donnelly, "Laser-engineered dissolving microneedle arrays for transdermal macromolecular drug delivery", Pharmaceut. Res. 28(8), 1919 (2011). http://dx.doi.org/10. 1007/s11095-011-0419-4

[32] S. Aoyagi, H. Izumi, Y. Isono, M. Fukuda and H. Ogawa, "Laser fabrication of high aspect ratio thin holes on biodegradable polymer and its application to a microneedle", Sensors and Actuators A: Physical. 139(1-2), 293-302 (2007). http://dx.doi.org/10. 1016/j.sna. 2006.11.022

[33] S. Liu, M.-n. Jin, Y.-s. Quan, F. Kamiyama, H. Katsumi, T. Sakane and A. Yamamoto, "The development and characteristics of novel microneedle arrays fabricated from hyaluronic acid, and their application in the transdermal delivery of insulin", J. Control Release. 161(3), 933-941 (2012). http://dx.doi.org/10. $1016 / j \cdot j$ conrel . 2012.05.030

[34] Y. Ito, M. Hirono, K. Fukushima, N. Sugioka and K. Takada, "Two-layered dissolving microneedles formulated with intermediate-acting insulin", Int. J. Pharm. 436(1-2), 387-393 (2012). http://dx.doi. org/10.1016/j.ijpharm. 2012.06.047

[35] J.-H. Park, M. G. Allen and M. R. Prausnitz, "Biodegradable polymer microneedles: fabrication, mechanics and transdermal drug delivery", J. Control Release 104(1), 51-66 (2005). http://dx.doi.org/10. 1016/j.jconrel. 2005.02.002

[36] J. W. Lee, J.-H. Park and M. R. Prausnitz, "Dissolving microneedles for transdermal drug delivery", Biomaterials 29(13), 2113-2124 (2008). http://dx.doi.org/ 10.1016/j.biomaterials. 2007.12.048

[37] M. C. Chen, S. F. Huang, K. Y. Lai and M. H. Ling, "Fully embeddable chitosan microneedles as a sustained release depot for intradermal vaccination", Biomaterials 34, 3077-3086 (2013). http://dx.doi.org/ 10.1016/j.biomaterials. 2012.12.041

[38] M. H. Ling and M. C. Chen, "Dissolving polymer microneedle patches for rapid and efficient transdermal delivery of insulin to diabetic rats", Acta Biomaterialia 9, 8952-8961 (2013). http://dx.doi.org/10.1016/j. actbio.2013.06.029

[39] M.-C. Chen, M.-H. Ling, K.-Y. Lai and E. Pramudityo, "Chitosan Microneedle Patches for Sustained Transdermal Delivery of Macromolecules", Biomacromolecules 13(12), 4022-4031 (2012). http://dx.doi. org/10.1021/bm301293d

[40] M. Kim, B. Jung and J.-H. Park, "Hydrogel swelling as a trigger to release biodegradable polymer microneedles in skin", Biomaterials 33(2), 668-678 (2012). http://dx.doi.org/10.1016/ j.biomaterials. 2011.09.074

[41] L. Y. Chu and M. R. Prausnitz, "Separable arrowhead microneedles", J. Control Release 149(3), 242249 (2011). http://dx.doi.org/10.1016/j.jconrel. 2010.10 .033

[42] R. F. Donnelly, T. R. R. Singh, M. J. Garland, K. Migalska, R. Majithiya, C. M. McCrudden, P. L.
Kole, T. M. T. Mahmood, H. O. McCarthy and A. D. Woolfson, "Hydrogel-forming microneedle arrays for enhanced transdermal drug delivery", Adv. Funct. Mater. 22(23), 4879-4890 (2012). http://dx.doi.org/ 10. 1002 /adfm. 201200864

[43] R. F. Donnelly, R. Majithiya, T. R. R. Singh, D. I. J. Morrow, M. J. Garland, Y. K. Demir, K. Migalska, E. Ryan, D. Gillen, C. J. Scott and A. D. Woolfson, "Design, optimization and characterisation of polymeric microneedle arrays prepared by a novel laser-based micromoulding technique", Pharmaceut. Res. 28(1), 41-57 (2010).http://dx.doi.org/10. 1007/s11095-010-0169-8

[44] R. Boehm, P. Miller, S. Hayes, N. Monteiro-Riviere and R. Narayan, "Modification of microneedles using inkjet printing", AIP advances 1(2), 022139-13 (2011). http://dx.doi.org/10.1063/1.3602461

[45] D. Yang, Y. Li and J. Nie, "Preparation of gelatin/PVA nanofibers and their potential application in controlled release of drugs", Carbohyd. Polym. 69(3), 538-543 (2007). http://dx.doi.org/10.1016/ j.carbpol.2007.01.008

[46] K. Masanori, J. Toguchida and M. Oka, "Preliminary study of polyvinyl alcohol-hydrogel (PVA-H) artificial meniscus", Biomaterials 24(4), 639-647 (2003). http://dx.doi.org/10.1016/ S0142-9612(02) 00378-2

[47] S. Yang, Y. Feng, L. Zhang, N. Chen, W. Yuan and T. Jin, "A scalable fabrication process of polymer microneedles", Int. J. Nanomed. 7, 1415 (2012). http:// dx. doi.org/10.2147/IJN. S28511

[48] J. Su, J. Mazzeo, N. Subbarao and T. Jin, "Conference report: pharmaceutical development of biologics: fundamentals, challenges and recent advances", Therapeutic Delivery 2(7), 865-871 (2011). http://dx.doi. org/10.4155/tde.11.58

[49] Y. K. Demir, Z. Akan and O. Kerimoglu, "Sodium alginate microneedle arrays mediate the transdermal delivery of bovine serum albumin", PloS one 8(5), e63819 (2013). http://dx.doi.org/10.1371/journal.pone. 0063819

[50] Y. K. Demir, Z. Akan and O. Kerimoglu, "Characterization of polymeric microneedle arrays for transdermal drug delivery", PloS one 8(10), e77289 (2013). http:// dx.doi.org/10.1371/journal . pone.0077289

[51] M. J. Garland, E. Caffarel-Salvador, K. Migalska, A. D. Woolfson and R. F. Donnelly, "Dissolving polymeric microneedle arrays for electrically assisted transdermal drug delivery", J. Control Release 159(1), 5259 (2012). http://dx.doi.org/10.1016/j.jconrel. 2012.01.003

[52] H. Chen, H. Zhu, J. Zheng, D. Mou, J. Wan, J. Zhang, T. Shi, Y. Zhao, H. Xu and X. Yang, "Iontophoresis-driven penetration of nanovesicles through microneedle-induced skin microchannels for enhancing transdermal delivery of insulin", J. Control Release 139(1), 63-72 (2009). http://dx.doi. org/10.1016/j.jconrel.2009.05.031

[53] M. G Nava-Arzaluz, I. Calderon-Lojero, D. QuintanarGuerrero, R. Villalobos-Garcia and A. Ganem- 
Quintanar, "Microneedles as transdermal delivery systems: combination with other enhancing strategies", Current Drug Delivery 9(1), 57-73 (2012). http://dx . doi.org/10.2174/156720112798376078

[54] A.-R. Denet, R. Vanbever and V. Préat, "Skin electroporation for transdermal and topical delivery", Adv. Drug Deliver. Rev. 56(5), 659-674 (2004). http://dx. doi .org/10.1016/j . addr. 2003.10.027

[55] V. Kumar, "Modulated iontophoretic delivery of small and large molecules through microchannels", Int. J. Pharm. 434(1-2), 106 (2012). http://dx.doi.org/10. 1016/j.ijpharm. 2012.05.030

[56] C.-J. Ke, Y.-J. Lin, Y.-C. Hu, W.-L. Chiang, K.-J. Chen, W.-C. Yang, H.-L. Liu, C.-C. Fu and H.-W. Sung, "Multidrug release based on microneedle arrays filled with pH-responsive PLGA hollow microspheres", Biomaterials 33(20), 5156-5165 (2012). http://dx. doi.org/10.1016/j.biomaterials. 2012.03.056

[57] X.-P. Fu, "On the Influence of the Psychological Shift of the Scholars in Mid-Ming Dynasty on Zisha Teapot Prosperity", Journal of Chengdu University of Technology (Social Sciences) 16(1), 11-15 (2008).

[58] J. Wu, T. Hou, M. Zhang, Q. Li, J. Wu, J. Li and Z. Deng, "An analysis of the chemical composition, performance and structure of China Yixing Zisha pottery from 1573 AD to 1911 AD", Ceram. Int. 39(3), 2589-2595 (2013). http://dx.doi.org/10. $1016 / j$.ceramint. 2012.09.021
[59] J. Sun and M. L. Ruan, "Microstructure and properties of Yixing Zisha ware", China Ceramics 4, 21-25 (1993).

[60] R. F. Donnelly, T. R. R. Singh, A. Z. Alkilani, M. T. C. McCrudden, S. O'Neill, C. O'Mahony, K. Armstrong, N. McLoone, P. Kole and A. D. Woolfson, "Hydrogelforming microneedle arrays exhibit antimicrobial properties: Potential for enhanced patient safety", Int. J. Pharm. 451(1-2), 76-91 (2013). http://dx.doi.org/ 10.1016/j.ijpharm. 2013.04.045

[61] R. F. Donnelly, T. R. R. Singh, M. J. Garland, K. Migalska, R. Majithiya, C. M. McCrudden, P. L. Kole, T. M. Mahmood, H. O. McCarthy and A. D. Woolfson, "Hydrogel-forming microneedle arrays for enhanced transdermal drug delivery", Adv. Funct. Mater. 22(23), 4879-4890 (2012). http://dx.doi.org/ 10.1002/adfm. 201200864

[62] K. Juntanon, S. Niamlang, R. Rujiravanit and A. Sirivat, "Electrically controlled release of sulfosalicylic acid from crosslinked poly(vinyl alcohol) hydrogel", Int. J. Pharm. 356(1-2),1-11(2008). http://dx.doi. org/10.1016/j.ijpharm. 2007.12.023

[63] Y. Qiu, G. Qin, S. Zhang, Y. Wu, B. Xu and Y. Gao, "Novel lyophilized hydrogel patches for convenient and effective administration of microneedlemediated insulin delivery", Int. J. Pharm. 437(1-2), 5156 (2012). http://dx.doi.org/10.1016/j.ijpharm. 2012.07 .035 